\title{
Poder, conocimiento y ciencias sociales
}

César GERMANÁ CAVER0

\begin{abstract}
RESUMEN
La conferencia inaugural aborda aspectos centrales sobre la problemática del poder, el conocimiento y las ciencias sociales en una perspectiva de pasado, presente y futuro. Reflexión que contribuya al necesario debate de la enseñanza de nuestras disciplinas.
\end{abstract}

Palabras clave: Poder, conocimiento, ciencias sociales, disciplinas, futuro.

\section{Power, knowledge and Social Sciences}

\section{ABSTRACT}

The inaugural conference addresses central aspects of the problem of power, knowledge and social sciences in a perspective of past, present and future. Reflection that contributes to the necessary debate on the teaching of our disciplines.

KEYWORDS: Power, knowledge, social sciences, disciplines, future. 


\section{$\mathrm{P}$} ara mí es un considerable honor pronunciar la clase inaugural del año académico 2015 de la Facultad de Ciencias Sociales. Primero, porque me siento comprometido con las ciencias sociales en nuestra Universidad, inicialmente como alumno y después como profesor. En segundo lugar, porque es un inapreciable privilegio impartir mi última clase para una audiencia compuesta por alumnos y alumnas, principalmente ingresantes a las seis escuelas de nuestra Facultad, y por colegas con los que he compartido durante muchos años numerosos proyectos e ilusiones para la renovación de nuestra Facultad y de nuestra Universidad.

En esta clase, me gustaría proponer algunas reflexiones, que han surgido como producto de los diversos cursos que he tenido a mi cargo en la Facultad, sobre el pasado, el presente y el futuro de las Ciencias Sociales y que pueden contribuir al necesario debate sobre la enseñanza de nuestras disciplinas.

\section{La construcción histórica de las ciencias sociales}

Como señala el Informe de la Comisión Gulbenkian para la reorganización de las ciencias sociales (Wallerstein, 1996), estas disciplinas surgieron en cuatro países de Europa y en Estados Unidos durante la segunda mitad del siglo XIX y en gran parte del siglo Xx se convirtieron en la forma legítima - esto es, científica - del conocimiento sobre la existencia social de los seres humanos. Este hecho nos plantea algunas cuestiones que me gustaría explorar brevemente.

\section{El carácter históricamente determinado del saber y del conocer}

Una primera observación radica en la tesis de que no existe una forma de pensamiento universalmente válido pues todas las maneras de conocer están ancladas en las condiciones históricas y sociales donde se han producido. Cada sistema histórico crea sus propias estructuras de saber/conocer, esto es, los códigos básicos que rigen el lenguaje, los esquemas de percepción y apreciación, los valores y las formas de racionalidad que constituyen el mundo intersubjetivo y que se fija en cada uno de los seres humanos, en la medida en que toda subjetividad es siempre intersubjetiva.

Esta es la razón que explica por qué las ciencias sociales no siempre han existido. Lo que se encuentra a lo largo de la historia de la humanidad es alguna forma de saber sobre la vida de los seres humanos en sociedad. Cada sistema histórico ha buscado explicar su origen, sus formas de organización social, incluyendo la justificación de la desigualdad social. Las religiones, los mitos,

\section{6/ REVISTA DE SOCIOLOGÍA 25}


las leyendas y, de manera más rigurosa, la filosofía, tal como surge en la Grecia clásica, han tratado de dar cuenta de la existencia social de los seres humanos.

Lo que conocemos como ciencias sociales constituyen un producto de un sistema histórico específico: el patrón de poder colonial $/$ moderno $^{1}$-la forma en la que se organizó la existencia social que surgió en 1492 y que actualmente está en crisis. De allí que el surgimiento de las ciencias sociales lleva la impronta, la huella, de las estructuras de saber/conocer eurocéntricas.

\section{El eurocentrismo y sus rasgos más relevantes}

El eurocentrismo puede ser considerado como un modo particular de plantear preguntas y ordenar las respuestas a la realidad tanto natural como históricosocial. Se trata de una forma específica de saber y de conocer que se origina en Europa y se impone en todo el planeta como la única forma legítima de pensar y conocer; constituye, por tanto, un universalismo europeo (Wallerstein, 2007), esto es, una visión provinciana que se considera universal.

En el patrón de poder colonial/moderno, el eurocentrismo se convierte en la única forma legítima de saber marginando, subalternizando o destruyendo las estructuras de saber de los pueblos colonizados. Los saberes que durante milenios se habían desarrollado en esos pueblos y habían servido de fundamento a sus específicas formas existencia social son violentamente relegados y reprimidos hasta el extremo de que sus propios portadores buscan desprenderse de ellos porque también los consideran inferiores.

Tres rasgos principales caracterizan a la forma de saber y de conocer eurocéntricas.

a) En primer lugar, la creencia en el principio de la simplificación. Según el planteamiento de Descartes, para conocer lo complejo es necesario dividirlo en tantas partes como sea posible para estudiar cada una de esas partes de manera aislada. Este principio ha llevado a la disyunción - separar lo que está junto - y a la reducción - explicar el conjunto por uno de sus elementos (Morin, 2007). La consecuencia de esta perspectiva es el predominio del dualismo epistemológico - sujeto/objeto, mente/ cuerpo, naturaleza/sociedad - y la creciente compartimentalización del saber, la especialización del conocimiento y el surgimiento de las disciplinas como categorías intelectuales, pues cada una tiene su propio objeto y método de estudio, y como categorías institucionales, al convertirse en 
departamentos que constituyen la base organizativa de las universidades modernas (Wallerstein, 2001).

b) En segundo lugar, la creencia en el principio de la estabilidad de los sistemas sociales y naturales. Se concibe la realidad como un mundo ordenado que funciona según leyes simples que es posible conocer; y a partir de ese conocimiento se puede prever lo va a ocurrir y, por lo tanto, controlar tanto el mundo natural como el mundo social. La consecuencia de esta creencia lleva al determinismo y a la reversibilidad; esto es, la eliminación de la historia como proceso creativo.

c) En tercer lugar, la creencia en el principio de la objetividad; principio según el cual se puede conocer la realidad tal como es, poniendo entre paréntesis al sujeto. La consecuencia de esta creencia es la aceptación de una ciencia libre de valores.

3. Consecuencias de las estructuras de saber/conocer en la construcción histórica de las ciencias sociales

a) La disciplinarización del conocimiento de vida social. Se simplifica la realidad histórico-social y cada una de las partes en la que se la ha dividido se convierte en el objeto de una determinada disciplina. Tres simplificaciones se encuentran en la base de la disciplinarización del conocimiento social. La primera simplificación: la separación entre la ciencia que estudia el mundo europeo - civilizado - y el mundo no europeo - no civilizado-; el mundo no europeo es estudiado por la antropología cuyo objeto es el de los pueblos cazadores y recolectores - “nuestros contemporáneos primitivos” como se titula un manual de antropología - y los estudios orientales u orientalismo que estudia a las grandes civilizaciones (china, indú, árabe) a las que se considera que han quedado inmovilizadas en el tiempo. La segunda simplificación: en el estudio de las sociedades europeas, la separación entre el estudio del pasado (la historia) y el estudio del presente. La tercera simplificación, la separación - de acuerdo a la ideología liberal- entre mercado (economía), estado (ciencia política) y sociedad civil (sociología). Además, las disciplinas sociales no solo tienen un objeto y un método para estudiar un ámbito específico de la realidad histórico-social, sino que se institucionalizan y forman los departamentos de la universidad moderna. La universidad moderna es una institución organizada en disciplinas (los departamentos); donde varios departamentos afines forman una facultad y el conjunto de facultades, la universidad (Wallerstein, 1996).

\section{8/ REVISTA DE SOCIOLOGÍA 25}


b) La neutralidad valorativa de la ciencia. Se considera que el científico se ocupa únicamente por buscar la verdad o falsedad de sus conocimientos pero no se interesa por otros valores: los valores éticos prácticos (el bien, la justicia) o estéticos (la belleza). Max Weber (1864-1920), uno de los padres fundadores de la ciencia social, sostuvo esta posición de una manera muy precisa. En una conferencia, en los últimos años de su vida, "La ciencia como profesión-vocación”, sostuvo: “Así como los helenos ofrecían sacrificios a Afrodita o a Apolo y, sobre todo, a los dioses de la propia ciudad, así también sucede hoy, aunque el culto se haya desmitificado y carezca de la plástica mítica que tenía en su forma original. Sobre esos dioses y su eterna contienda decide el destino pero no, por cierto, la ciencia" (Weber, 1966). El mundo se ha desencantado y, como señala el mismo Weber, "una cosa puede ser verdadera aun cuando no sea bella, ni santa, ni buena". El positivismo - corriente dominante durante mucho tiempo en las ciencias sociales - aparece como la mejor expresión de esta manera de enfocar el examen de la vida social. Esta corriente se limita a comprobar los vínculos causales regulares entre los fenómenos, el examen de lo positivo, la realidad tal como es, pero abandona toda reflexión sobre la realidad como debe ser. El positivismo acepta el ser como el deber ser; de allí su carácter conservador pues se prohíbe un examen crítico de la organización social realmente existente al no comparar lo que es con lo que debe ser, por ejemplo, una sociedad justa, democrática e igualitaria.

\section{La crisis de las ciencias sociales}

Hacia la década de 1970, todo el edificio del conocimiento social que se había construido en los últimos cien años empieza de erosionarse y se inicia un período marcado por la crisis de las ciencias sociales, etapa en la que todavía nos encontramos ${ }^{2}$. Esta crisis es parte de una crisis mayor: la de toda la estructura de poder que ha dominado el mundo en los últimos quinientos años. Es la crisis del capitalismo, del Estado-nación, del patriarcalismo, del medio ambiente. Todo el sistema histórico que surgió en 1492 se ha venido erosionando y hemos entrado a un período de caos sistémico (Wallerstein, 1998).

2 Michel Wieviorka, en el discurso inaugural, como presidente de la Asociación Internacional de Sociología, en el XVII Congreso Mundial de Sociología de julio de 2010, sostuvo: "La ciencias sociales, y en particular la sociología, han entrado en una fase de mutación: están saliendo de la era clásica, y muchos pensamos que esta salida empezó a finales de los años sesenta o inicios de los setenta”. 
De manera precisa, cuando hablamos de crisis nos referimos a la existencia de problemas que el sistema no puede resolver (se trata de crisis estructural, sistémica); el sistema no puede seguir reproduciéndose y llega a un punto de bifurcación a partir del cual se abre una etapa caótica de la que surgirá otro u otros sistemas que todavía no podemos conocer.

En el caso de las ciencias sociales han surgido problemas o desafíos que esta forma de conocimiento de la realidad histórico-social no ha podido resolver y ha llevado a la necesidad de replantear o abandonar las premisas sobre las que construyeron históricamente estas disciplinas. Quiero examinar tres desafíos que considero fundamentales para comprender la crisis de las ciencias sociales.

a) El primer desafío está dado por la emergencia de la epistemología de la complejidad. Esta epistemología parte del reconocimiento de la existencia de sistemas con un gran número de elementos interactivos que se interdefinen y se organizan en una totalidad de componentes heterogéneos y de naturaleza histórica. Una epistemología de esta naturaleza conlleva la superación de las "disciplinas" y apunta a la unificación del saber en una orientación transdisciplinaria, perspectiva que apunta a -como señala Pablo González Casanova - "volverse especialista en el estudio de un problema, independientemente de que la especialización signifique manejar disciplinas que se enseñen en diferentes facultades" (González Casanova, 2005).

Las tesis básicas de esta epistemología han sido desarrolladas en las ciencias naturales, particularmente en la física, sanctus sanctorum del saber hegemónico. El punto de partida de esta manera de conocer es el señalamiento del "fin de las certidumbres", indicado por llya Prigogine (Prigogine, 1997). La ciencia ya no se identifica con la certidumbre: "Tanto en la dinámica clásica como en la física cuántica - escribe Prigogine - las leyes fundamentales ahora expresan posibilidades, no certidumbres" y agrega: "Hoy creemos estar en un punto crucial de esa aventura, en el punto de partida de una nueva racionalidad que ya no identifica ciencia y certidumbre, probabilidad e ignorancia" (Prigogine, 1997: 11 y 12). La ciencia clásica privilegió la estabilidad y el orden, eliminando la incertidumbre; en cambio, la nueva ciencia privilegia las fluctuaciones y la inestabilidad. En este sentido se puede afirmar que, como lo plantean Nicolis y Prigogine: "desde la década de los años sesenta, somos testigos de una revolución en las ciencias matemáticas y físicas que nos obliga a adoptar una postura completamente nueva para la descripción de la naturaleza" (Nicolis y

\section{0/ REVISTA DE SOCIOLOGGIA 25}


Prigogine, 1997: 22). Y con mayor razón para la descripción de la realidad histórico-social.

b) Un segundo desafío está dado por el cuestionamiento al eurocentrismo de las ciencias sociales. En la periferia del actual patrón de poder moderno/colonial han surgido diversas corrientes de pensamiento que se han planteado la necesidad de lograr la descolonización de las ciencias sociales. Dos ejemplos claros se encuentran en las llamadas corrientes poscoloniales: de una lado, la crítica al orientalismo que realizó Eduard Said (2003) al considerarlo un discurso colonial - sistema de creencias, conocimientos y representaciones en todas las esferas de la cultura occidental que mostrarían su superioridad frente al oriente - que permite legitimar la dominación imperialista; y de otro lado, los estudios subalternos de los historiadores hindúes que tenían como objetivo "producir análisis históricos en que los grupos subalternos fueran vistos como sujetos de la historia"; es decir, estudiar la historia desde la India y no desde Europa (Chakrabarty, 2008) .

c) Un tercer desafío está dado por la perspectiva de análisis de la colonialidad/ descolonialidad del poder (Quijano, 1991, 1993, 200a, 2000b, 2000c, 2000d). El análisis de la colonialidad/descolonialidad del poder establece una perspectiva de conocimiento - una manera de percibir la realidad, de producir las preguntas y ordenar las respuestas sobre la existencia social de los seres humanos - que en aspectos fundamentales permite abrir muchas cuestiones importantes que el pensamiento eurocéntrico había cerrado. Ha surgido como un desafío a las formas eurocéntricas de producir conocimientos porque cuestiona los fundamentos sobre los que se construyeron las estructuras del saber hegemónicas en el sistema moderno/colonial capitalista.

Siguiendo la teóricamente innovadora propuesta de Aníbal Quijano, se puede señalar que con la conquista europea, en 1492, de lo que posteriormente se va a llamar América, surgió un patrón de poder sui géneris que tiene como características la colonialidad y la modernidad. De un lado, la colonialidad en la medida en que las relaciones de poder que se establecen con la conquista están atravesadas por la idea de "raza" como cimiento de la clasificación social de los seres humanos; esto es, la consideración de que los conquistadores se imaginaban seres humanos superiores y se autoidentificaban como "blancos", mientras que a los conquistados los miraban como humanamente inferiores y los identificaban como "indios" y “negros”. La idea de "raza”, al naturalizar una relación social, buscará legiti- 
mar la dominación y explotación de la población originaria y de los esclavos africanos y será parte fundamental del mantenimiento del patrón de poder aún después de producida la independencia política de las colonias españolas y portuguesas pues permite garantizar que dominadores y dominados acepten como natural la dominación. De otro lado, la modernidad, la otra cara de la colonialidad, se refiere a la creciente racionalización instrumental de la vida social, donde el avance científico-tecnológico se presenta como su principal indicador.

\section{El futuro de las ciencias sociales. ¿Hacia dónde van las ciencias sociales?}

Con seguridad, los científicos sociales no somos futurólogos, aunque muchos no desdeñen desempeñar ese papel. El futuro es por naturaleza incierto, sobre todo si no se asume la perspectiva determinista que ha dominado a la ciencia social, tanto la marxista como la no marxista. Y la incertidumbre es aún mayor cuando se vive en un período de transición como en el que nos encontramos, donde toda la estructura de poder que se constituyó hace quinientos años se está desmoronando y otra, que todavía no conocemos, está surgiendo delante de nosotros. Nos hallamos en una época donde - como con tanta precisión señala Immanuel Wallerstein - “[...] el sistema-mundo moderno se aproxima a su fin y está ingresando en una era de transición hacia un sistema histórico nuevo, cuyos contornos no conocemos ahora - no podemos conocerlos por anticipado - , pero cuya estructura podemos ayudar a modelar" (Wallerstein, 2002: 63).

Con mayor razón, nuestras posibilidades de identificar el futuro de las ciencias sociales son más inciertas. Las diversas perspectivas que han surgido o están surgiendo en el campo de las ciencias sociales se disputan la hegemonía para lograr que se les considere como la forma de conocimiento socialmente legítimo sobre la vida social. Y el futuro se definirá por el resultado de esas luchas teóricas y prácticas para lo cual se movilizan diversas formas de capital, como las denomina Pierre Bourdieu, desde los recursos económicos hasta los simbólicos.

Me gustaría explorar los interrogantes que plantea la elección de cuál es el camino posible y deseable por el que debería transitar la ciencia social. Se trata, fundamentalmente, de elegir racionalmente entre varios fines; esto es, lo que Max Weber llamó “racionalidad material”. Es una cuestión donde se busca responder a la pregunta sobre lo que debe ser la ciencia social: ¿Cuál es el mejor modelo de ciencia social que queremos construir?

\section{2/ REVISTA DE SOCIOLOGGIA 25}


En primer lugar, necesitamos una ciencia social crítica. Esta perspectiva de análisis surge en Europa en los siglos xIx y xx como consecuencia del profundo malestar e inconformismo de las clases explotadas y dominadas por el capital respecto al incumplimiento de las principales promesas de la modernidad: el logro de la igualdad, de la libertad y la solidaridad. Para Boaventura de Sousa Santos, el pensamiento crítico es aquel que "no reduce la 'realidad' a lo que existe" sino que, más bien, la considera como "un campo de posibilidades" y su tarea consiste en "definir y ponderar el grado de variación que existe más allá de lo empíricamente dado" (Santos, 2003: 26). A diferencia de las ciencias sociales celebratorias de la modernidad y del capitalismo, que asumen la "neutralidad valorativa" del científico social, el pensamiento crítico tiene una clara orientación ética y normativa. No acepta al ser como el deber ser, según la propuesta de la sociología positivista, desde A. Comte a T. Parsons, sino que se plantea la imperiosa exigencia de la transformación del ser por obra del deber ser. Este sentido del pensamiento crítico lo habían señalado con precisión Th. Adorno y M. Horkheimer: "La ciencia puede ser algo más que la simple duplicación de lo real en el pensamiento, sólo si está impregnada del espíritu de la crítica. Explicar la realidad significa siempre romper el cerco mágico de la duplicación. Crítica no significa aquí subjetivismo, sino confrontación de la cosa con su propio concepto. Lo dado se ofrece sólo a una visión que lo considere desde el punto de vista de un verdadero interés: de una sociedad libre, de un Estado justo, del desarrollo de lo humano. Y el que no compara las cosas humanas con lo que ellas quieren significar las ve, en definitiva, en forma no sólo parcial, sino, además, falsa" (Adorno y Horkheimer, 1971: 22). Una ciencia social que se oriente en el sentido de la tradición del pensamiento crítico $-\mathrm{y}$ que supone el desmantelamiento del mito de la "neutralidad valorativa"-, debe reflexionar simultáneamente en términos intelectuales, morales y políticos.

Sin embargo, el pensamiento crítico no sería fructífero si no se planteara la tarea de la descolonialidad del saber, esto es, el cuestionamiento radical a la hegemonía eurocéntrica. Esta empresa se convierte en el mayor desafío para lograr una profunda reestructuración de la ciencia social que contribuya de manera efectiva a la lucha por alcanzar una sociedad más democrática y más igualitaria. De esta manera, se estarían dando las condiciones para la emergencia de una perspectiva de conocimiento alternativa a la del eurocentrismo. A diferencia del período inmediatamente anterior, donde las derrotas del movimiento obrero y popular trajo como consecuencia que se fuera extinguiendo el horizonte utópico en el imaginario histórico, cuyo núcleo 
principal era crítico, y por lo tanto, también, el pensamiento social crítico, en el período actual, el tiempo de la derrota parece estar llegando a su fin. Con el nuevo referente social en las fuerzas cuestionadoras del sistema, aparecen las condiciones para la descolonialidad de las estructuras del saber/conocer.

En segundo lugar, se debe trabajar para superar los estrechos límites que han fracturado las ciencias sociales en disciplinas, que las convirtieron en compartimentos estancos. Se trata de la búsqueda de una creciente unificación de las disciplinas sociales. Esta perspectiva transdisciplinaria permitiría dar cuenta de la complejidad de la vida social, pues la ultraespecialización que las ciencias sociales han estado padeciendo ha sido particularmente improductiva. En este sentido, el reto de las ciencias sociales radica en la necesidad de construir una ciencia social, donde la especialización sea trabajada por problemas y no como campos arbitrariamente definidos. Las segmentaciones con las que se constituyeron las ciencias sociales - europeocivilizado/no-europeo-no civilizado, pasado/presente y mercado/estado/ sociedad civil - no tienen en la actualidad ningún fundamento epistemológico o teórico válido. La epistemología de la complejidad, la teoría de los sistemas y la mundialización de las relaciones sociales lleva necesariamente a que solamente podamos comprender cabalmente la sociedad humana desde una perspectiva holística, totalizante.

En tercer lugar, una ciencia social crítica no eurocéntrica debe superar el insostenible dualismo del saber europeo que se estructuró alrededor de la tesis de las "dos culturas": la científica y la humanística. Esta tesis fue planteada inicialmente por C. P. Snow, en 1959, en una conferencia donde sostuvo la existencia, en el mundo occidental, de "dos grupos polarmente antitéticos: en un polo tenemos los intelectuales literarios [...] y en el otro, los científicos"; cada uno de esos grupos tiene su propia cultura - "hábitos, supuestos y comunes maneras de vivir" - y "entre ambos grupos un abismo de incomprensión mutua” (Snow, 1977: 14). Como lo ha mostrado U. Cerroni, esta propuesta está profundamente enraizada en las estructuras del pensamiento moderno; pero es ajena al espíritu del Renacimiento, donde ambas culturas se adhieren al mismo programa intelectual. Sin embargo, a partir de esa época se irá perfilando el divorcio entre la visión humanista del mundo sociohistórico y la nueva ciencia experimental y "precisamente con Kant - propone Cerroni- parece cristalizar definitivamente la escisión entre ciencias de la naturaleza y ciencias del hombre, en base a la cual trabaja desde hace dos siglos nuestra cultura como presupuesto ya consolidado" (Cerroni, 1971: 17). Y este dualismo, ciencia y cultura, naturaleza y humanidad, aparece

\section{4/ REVISTA DE SOCIOLOGÍA 25}


como característica del saber moderno, del cual hace parte lo que I. Prigogine y I. Stengers denominan la "ciencia clásica", cuyo fundamento lo encuentran en la filosofía crítica de Kant. "No solamente el científico no puede conocer la cosa en sí - señalan Prigogine y Stengers - sino que las preguntas que pueden hacerse no tienen pertinencia alguna para los verdaderos problemas de la humanidad; ni la belleza, ni la libertad, ni la ética son objeto de conocimiento positivo, o sea de ciencia: pertenecen al mundo noumenal, dominio de la filosofía, totalmente extraño al mundo de los fenómenos" (Prigogine y Stengers, 2004: 123). Es posible argumentar que la tesis de las dos culturas que ha caracterizado a las estructuras del saber del sistema-mundo moderno es la consecuencia del "desencantamiento del mundo" al que se refería Max Weber cuando señalaba que la modernidad había diferenciado las diferentes esferas de valor: "si hay algo que nosotros sabemos es que una cosa puede ser santa aun cuando no sea bella y también por el hecho y en la medida en que no es bella [...] y que una cosa puede ser bella aun cuando no sea buena [...] Y finalmente, es una verdad de todos los días que una cosa puede ser verdadera aun cuando no sea bella, ni santa, ni buena" (Weber, 1966: 26). La consecuencia de esta perspectiva de conocimiento ha sido una enorme limitación en la forma de examinar la realidad: mientras que la filosofía y las humanidades tenían por objetivo la búsqueda de lo bueno y bello; la ciencia se atribuía el monopolio de la búsqueda de la verdad mediante la investigación experimental de la realidad.

Del enfrentamiento de las ciencias sociales con las "dos culturas" surgió la disputa metodológica que se dio a fines del siglo xıx. De un lado, estaban quienes querían arrastrar a las ciencias sociales hacia las ciencias naturales y recurrieron al enfoque nomotético - la búsqueda de leyes universales-, como en el caso de la economía, la sociología y la ciencia política; de otro lado, los que se inclinaban por las humanidades y para ello recurrieron al enfoque idiográfico - la comprensión de fenómenos únicos e irrepetibles-, como en el caso de la historia. Desgarradas por este dualismo metodológico, las ciencias sociales no lograron su plena institucionalización. Sólo a fines del siglo xIX, con la hegemonía del positivismo y de la perspectiva nomotética fueron incorporadas al mundo académico como parientes atrasados de las ciencias naturales.

La ciencia social renovada debe convertirse en el lugar de encuentro de las esferas de valor que la modernidad europea occidental ha diferenciado. Como señala Wallerstein: "Lo que había sido un campo centrífugo de fuerzas en el mundo del conocimiento se ha convertido en uno centrípeta, y la ciencia 
social es ahora central al conocimiento. Estamos en el proceso de superar las "dos culturas", de tratar de reunir en un solo ámbito la búsqueda de lo verdadero, lo bueno y lo bello. Esto es una causa para regocijarse, pero será una tarea ardua de acometer" (Wallerstein, 2001: 292). Se busca romper el muro del conocimiento entre las humanidades y las ciencias. En este sentido, se puede señalar que estamos en el camino de alcanzar el reencantamiento del mundo, que la modernidad eurocéntrica había desencantado.

En cuarto lugar, la ciencia social necesita identificar ejes problemáticos que den cuenta de la nueva sociedad que magmáticamente está surgiendo delante de nosotros. El núcleo básico de esa problemática está dado por la investigación y el debate de la nueva estructura de poder que se está configurando en América Latina, su organización y sus tendencias de desarrollo. Ello implicará el estudio de la nueva heterogeneidad estructural, las nuevas formas de agrupamiento social que han surgido como consecuencia de la crisis de la estructura de clases del capital que estalló en los años setenta, la nueva institucionalidad política y la reconfiguración del Estado y la profunda reorganización del mundo intersubjetivo.

Soy consciente de que estas reflexiones son provisionales; pero he querido planteárselas en esta clase inaugural para incentivar la discusión sobre lo que hacemos, o lo que van a hacer los que recién se incorporan a nuestra Facultad. Después de todo, las ciencias sociales - como toda ciencia- no son sino un largo e interminable debate al cual todos estamos invitados a participar.

\section{Referencias bibliográficas}

Cerroni, Umberto (1971). Metodología y ciencia social. Barcelona: Martínez Roca. Chakrabarty, Dipesh (2008). Al margen de Europa. Pensamiento poscolonial y diferencia histórica. Barcelona: Tusquets.

González Casanova, Pablo (2005). Las nuevas ciencias y las humanidades. De la Academia a la Política. Barcelona: Anthropos Editorial.

HorkHeimer, Max y Adorno, Theodor (1971). La sociedad. Lecciones de sociología. Buenos Aires: Proteo.

Morin, Edgar (2007). Introducción al pensamiento complejo. Barcelona: Gedisa. NicolIS, Grégoire y PRIGOGINE, Ilya (1997). La estructura de lo complejo. En el camino hacia una nueva comprensión de las ciencias. Madrid: Alianza Editorial.

Prigogine, llya y Stengers, Isabelle (2004). La nueva alianza. Metamorfosis de la ciencia. Madrid: Alianza Editorial.

PRIGOGINE, llya (1997). El fin de las certidumbres. Madrid: Taurus. 
QuijAno, Aníbal (1991). “Colonialidad y modernidad/racionalidad”, en: Revista del Instituto Indigenista Peruano, Vol. 13, No. 29, Lima, pp. 11-20.

Quijano, Aníbal (1993). "Raza, etnia y nación en Mariátegui: cuestiones abiertas”, en: Aníbal Quijano et al., José Carlos Mariátegui y Europa. El otro aspecto del descubrimiento. Lima: Amauta, pp. 167-188.

QuijAno, Aníbal (2000a). “Colonialidad del poder, eurocentrismo y América Latina”, en: Edgardo Lander (comp.), Colonialidad del saber: eurocentrismo y ciencias sociales. Perspectivas latinoamericanas. Buenos Aires: Clacso/Unesco, pp. 201-246. QuijAno, Aníbal (200ob). “Colonialidad del poder, globalización y democracia”, en: Tendencias básicas de nuestra época. Caracas: Instituto de Altos Estudios Internacionales Pedro Gual, pp. 21-65.

QUIJANo, Aníbal (2000c). “Colonialidad del poder y clasificación social”, en: Journal of World-Systems Research, Vol. VI, No. 2, American Sociological Association, pp. 342-386.

Quijano, Aníbal (2001d). "Colonialidad, poder, cultura y conocimiento en América Latina”, en: Walter Mignolo (comp.), Capitalismo y geopolítica del conocimiento. El eurocentrismo y la filosofía de la liberación en el debate intelectual contemporáneo. Buenos Aires: Signo, pp. 117-131.

SAID, Edward (2003). Orientalismo. Barcelona: Debolsillo.

SAntos, Boaventura de Sousa (2003). La caída del Angelus Novus. Ensayos para una teoría social y una nueva práctica política. Bogotá: ILSA/UNC.

Snow, C. P. (1977). Las dos culturas y un segundo enfoque. Madrid: Alianza.

Wallerstein, Immanuel (Coordinador), (1996). Abrir las Ciencias Sociales. México: Siglo XXI.

WallersteIn, Immanuel (1998). Utopística o las opciones históricas del siglo XXI. México: Siglo XXI.

WALLERSTEIn, Immanuel (2001). Conocer el mundo, saber el mundo: el fin de lo aprendido. Una ciencia social para el siglo XXI. México: Siglo XXI.

WALlerstein, Immanuel (2002). Un mundo incierto. Buenos Aires: Libros del Zorzal. Wallerstein, Immanuel (2007). Universalismo europeo. El discurso del poder, México, Siglo XXI.

Weber, Max (1966). El sabio y la política. Córdova: EUDUCOR. 